\title{
Performance and track-based alignment of the Phase-1 upgraded CMS pixel detector
}

\author{
Valeria Botta*† \\ Deutsches Elektronen-Synchrotron (DE) \\ E-mail: valeria.botta@cern.ch
}

The Compact Muon Solenoid (CMS) detector is a multi-purpose detector constructed in order to study high-energy particle collisions at the Large Hadron Collider (LHC) at CERN. The all-silicon design of the tracking system of the CMS experiment provided excellent resolution for charged tracks and an efficient tagging of jets during Run 1 and Run 2 of the LHC. After the pixel detector of the CMS experiment was upgraded and installed during the shutdown in the beginning of 2017, the positions and orientations of the tracker modules needed to be determined with a precision of several micrometers. The alignment also needs to be quickly recalculated each time the state of the CMS magnet is changed between $0 \mathrm{~T}$ and $3.8 \mathrm{~T}$. The latest results of the CMS tracker performance in the 2017 run are presented, with a special focus on alignment and resolution performance using several million reconstructed tracks from cosmic rays and collision data.

The European Physical Society Conference on High Energy Physics

5-12 July

Venice, Italy

${ }^{*}$ Speaker.

${ }^{\dagger}$ On behalf of the CMS Collaboration. 


\section{Introduction}

The silicon pixel tracker is the innermost component of the CMS detector [1] and it is divided into the central barrel region (BPIX) and two forward endcaps (FPIX). During the LHC shutdown at the beginning of 2017, CMS entirely replaced its pixel detector with a new upgraded one [2], aiming at improving the tracking capabilities despite the foreseen increase of the instantaneous luminosity. With respect to the previous pixel detector, one barrel layer at high radius $(\mathrm{r}=16 \mathrm{~cm})$ and one additional disk in each endcap have been added. Moreover, the innermost barrel layer is located $15 \mathrm{~mm}$ closer to the interaction point, allowing a more precise extrapolation of the tracks to the interaction region. The pixel size is left unchanged and measures $100 \mu \mathrm{m} \times 150 \mu \mathrm{m}$.

A comparison of the tracking performance that can be achieved with the new pixel detector with respect to the previous one has been performed using simulated events [3]. It suggests an increased tracking efficiency and a reduction of the fake rate by roughly a factor 3 over the whole pseudorapidity $(\eta)$ range, as well as improvements in the track impact parameter and vertex resolutions by approximately 30\%, as shown in Figure 1. Precise measurements of the vertex positions and of the track impact parameters are crucial, e.g. to identify events with the presence of jets originating from the hadronisation of heavy flavour quarks. Such events are of primary interest in many physics analyses aiming at characterising the recently discovered Higgs boson or searching for new physics beyond the Standard Model. The expected improvement in tracking and vertex reconstruction due to the pixel upgrade should result in approximately $10 \%$ increase of the b-jet identification efficiency while keeping the same fake rate [4].
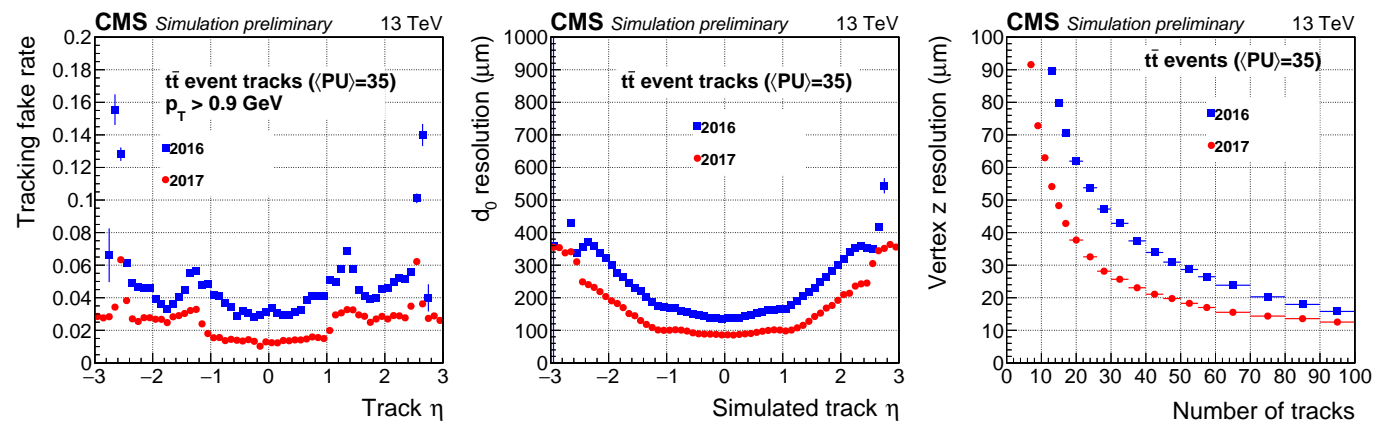

Figure 1: Comparison of the expected tracking performance for the old (2016) and the new (2017) pixel detector, estimated from simulation [3], in terms of track fake rate (left), track transverse impact parameter resolution (middle) and longitudinal vertex resolution (right).

The expected performance can only be achieved thanks to excellent hardware and calibrations of the CMS tracker. In particular, as tracks are reconstructed fitting a set of hits in the tracker modules, it is crucial to achieve the best possible hit resolution - that for silicon pixel modules is $\mathrm{O}(10 \mu \mathrm{m})$ - as well as to accurately determine the modules positions. In 2016, the latter could be measured with a precision of $\mathrm{O}(1 \mu \mathrm{m})$ for pixel modules [7] by employing track-based alignment.

\section{Track-based Alignment}

The aim of track-based alignment is to accurately determine the positions and orientations of the tracker modules, i.e. alignment parameters, from a large set of reconstructed tracks. As tracks 
passing through the same modules correlate alignment parameters, the latter can be determined by minimising the quadratic sum of the residuals between the measured and fitted track hits, based on the fact that such residuals are a measure of the misalignment. A detailed description of the algorithms used in CMS can be found elsewhere [5,6]. In the following, two coordinate systems are used: the global one, with the origin at the nominal collision point, the $x$-axis pointing to the centre of the LHC, the $y$-axis pointing up (perpendicular to the LHC plane), and the $z$-axis along the anticlockwise-beam direction, and the local one, defined for each module with the origin at the geometric centre of the active area of the module, the $x$-axis defined along the more precisely measured coordinate of the module, the $y$-axis orthogonal to the $x$-axis and in the module plane, pointing away from the readout electronics, and the $z$-axis normal to the module plane [5].

The alignment of the new pixel detector represented a big challenge, as some detector structures can be located even a few millimetres away from their nominal positions after the installation, and the new pixel detector was ready to record data only a few weeks before the beginning of the 2017 LHC physics run. In such commissioning phase, CMS recorded cosmic ray data, first at $0 \mathrm{~T}$ magnetic field and then at full magnetic field of $3.8 \mathrm{~T}$. The first alignments were performed using $0 \mathrm{~T}$ cosmic ray data. They aimed at deriving the correct position of the large mechanical structures - also called high level (HL) structures - of the pixel detector, corresponding to the two halves of the barrel pixel subdetector and of each pixel endcap. After that, the whole pixel detector was aligned down to the level of single modules by performing module level (ML) alignment. The largest measured corrections with respect to the geometry assumed before performing any alignment were a shift of about $3 \mathrm{~mm}$ of one of the the forward pixel endcaps in the longitudinal direction, and a $2 \mathrm{~mm}$ displacement of the whole pixel barrel subdetector in the horizontal plane, as shown in Figure 2.
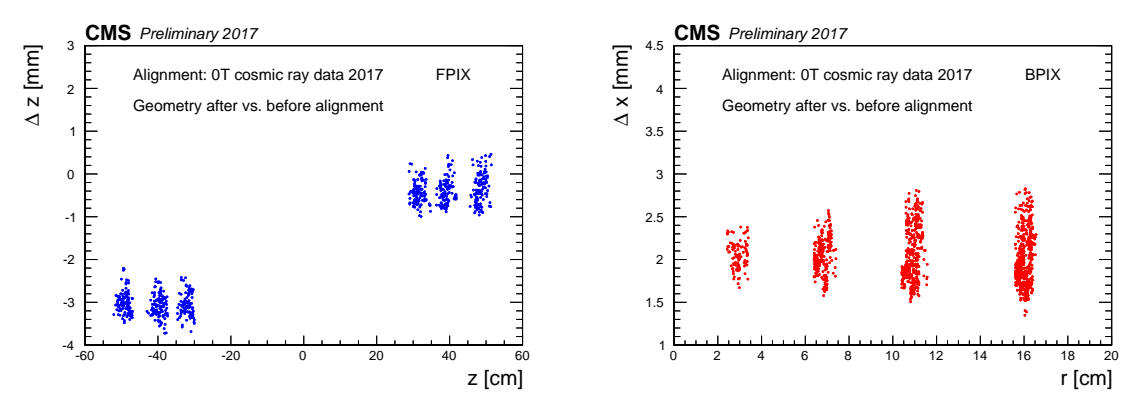

Figure 2: Difference of the modules positions after performing module-level alignment with respect to their assumed positions before performing any alignment. Every dot corresponds to a module. Left: difference $\Delta \mathrm{z}$ (after-before) alignment as a function of $z$. The FPIX $-z$ side is displaced by $-3 \mathrm{~mm}$ in the global- $z$ coordinate with respect to the position assumed before performing alignment, while the displacement of the $+z$ side is $-0.4 \mathrm{~mm}$. Right: difference $\Delta \mathrm{x}$ (after-before) alignment as a function of the radial coordinate $r$, for the barrel pixel modules. The barrel pixel is displaced by $+2 \mathrm{~mm}$ in the global $x$-coordinate with respect to the position assumed before performing alignment [8].

The improvement due to each alignment iteration can be evaluated in different ways, using quantities that are particularly sensitive to the chosen set of alignment parameters. Figure 3 shows the distributions of the residuals between the fitted and measured hit positions, in the local- $x$ direction [6], for FPIX (left) and BPIX (right) modules separately. In order to obtain an unbiased 

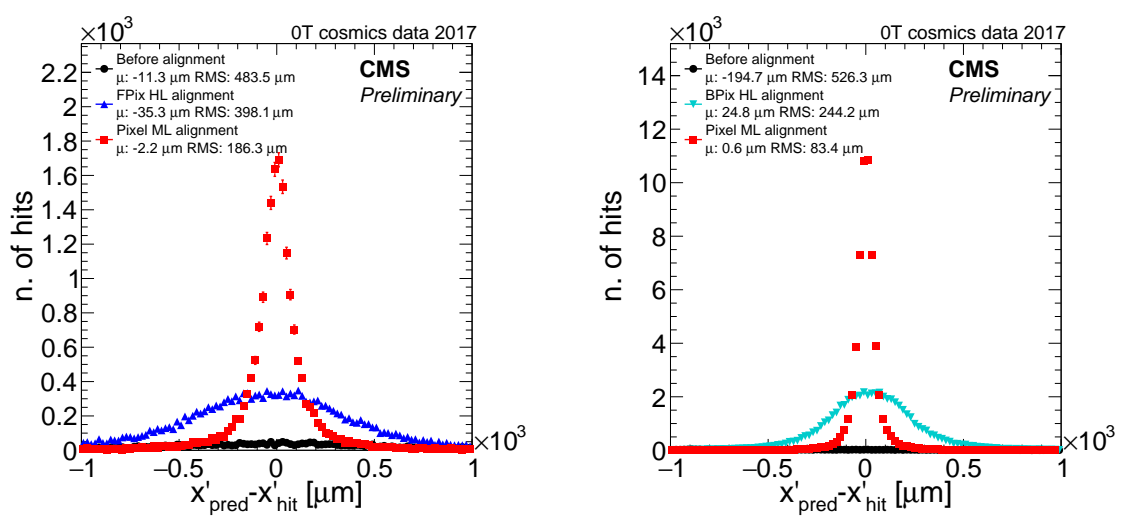

Figure 3: Unbiased track-hit residuals for $0 \mathrm{~T}$ tracks along the local- $x$ direction, for FPIX (left) and BPIX (right) modules. After the first alignment of the forward (blue triangles) and barrel (cyan triangles) pixel high-level structures both the bias and the width of the distributions are strongly reduced with respect to the geometry assumed before performing any alignment (black circles). The width of the distributions is further reduced after aligning the full pixel tracker down to the level of single modules (red squares). The mentioned alignments have been performed using $0 \mathrm{~T}$ cosmic ray tracks [8].

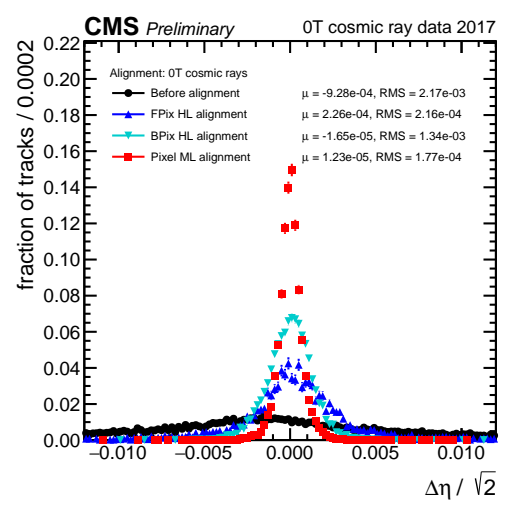

Figure 4: Normalised difference between the two halves of a cosmic ray track at $0 \mathrm{~T}$, split at the point of closest approach to the interaction region, in the track pseudorapidity $\eta$. After the first alignment of the barrel and forward pixel high-level structures (triangles) both the bias and the width of the distribution are strongly reduced with respect to the geometry assumed before performing any alignment (black circles). The width of the distribution is further reduced after aligning the full pixel tracker down to the level of single modules (red squares). The mentioned alignments have been performed using $0 \mathrm{~T}$ cosmic ray tracks [8].

measurement, the hit under consideration is removed from the track fit. The different curves show the distributions before performing any alignment (black dots), after the high-level structures alignment (blue and cyan triangles for FPIX and BPIX, respectively) and after the full module level alignment (red squares). After each alignment iteration, both the bias and the width of the distributions are considerably reduced, indicating respectively a reduction of systematic misalignment effects and an improvement of the local precision.

Another way to estimate the alignment performance is by means of the "track-split" validation. As cosmic rays cross the whole CMS detector, they can be split at the point of closest approach to the centre of the detector and the two track halves can be refitted independently. Track parameters 

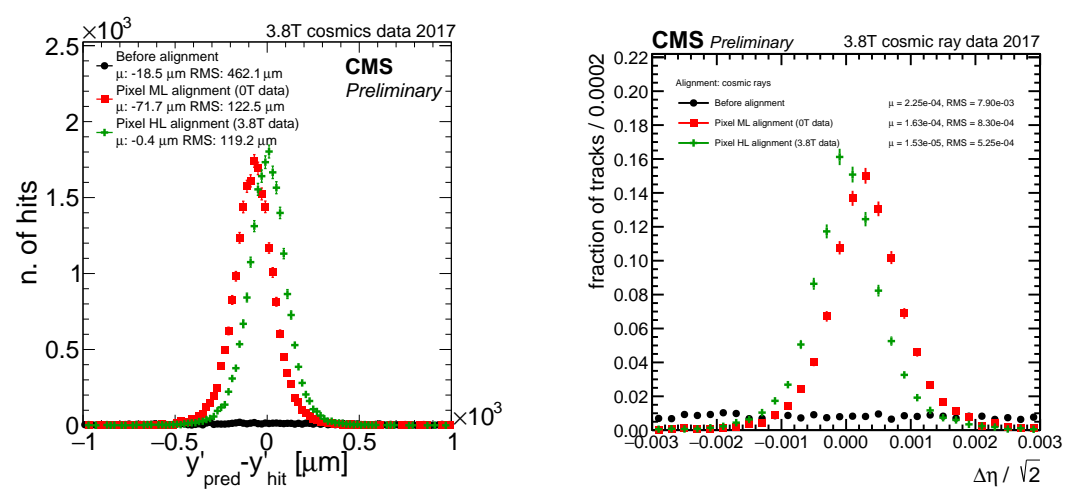

Figure 5: Unbiased track-hit residuals along the local-y direction for BPIX modules (left) and normalised $\Delta \eta$ between the two halves of a cosmic-ray track split at the point of closest approach to the interaction region (right), for cosmic ray tracks at 3.8 T. After the ramp up of the magnetic field, the positions of the pixel highlevel structures have been recomputed using $3.8 \mathrm{~T}$ cosmic ray tracks. The latter alignment (green crosses) shows improved performance over the geometry derived from $0 \mathrm{~T}$ cosmic ray data, and strongly reduces the bias of the distribution. As a comparison, the geometry assumed before performing any alignment is also shown (black circles) [8].

of the two halves that differ from each other hint at misalignments. Figure 4 shows the difference in the track pseudorapidity $\eta$ for such split cosmic ray tracks, after each alignment iteration. The improvement achieved after each alignment iteration can be observed, as both the bias and the width of the distributions are considerably reduced. A further alignment update was necessary after the CMS magnetic field was turned on, as the magnetic field change is known to induce sizeable movements of the pixel detector high-level structures. As a consequence, the geometry derived at $0 \mathrm{~T}$ became inadequate, showing biases in the track-hit residuals distributions and in the track-split validation (Figure 5, red squares). Therefore, a further alignment of the pixel detector was performed using cosmic ray data collected at $3.8 \mathrm{~T}$ and such biases could be successfully cured (Figure 5, green crosses).

\section{Hit Resolution}

The first measurements of the pixel hit resolution have been performed for barrel pixel modules of layers 2 and 3, using pp collision tracks with a transverse momentum $p_{T}>12 \mathrm{GeV}$ [9]. For such intermediate pixel layers the hit resolution can be estimated with the "triplet" method: tracks with pixel hits in layers 1,2 and 3 are refitted excluding the hit on layer 2, and the distribution of residuals between the measured and interpolated hit position from the refitted track is obtained. The standard deviation obtained from fitting the residual distribution provides an estimate of the hit resolution for layer 2. The measurement for layer 3 is done similarly, considering tracks with pixel hits on layers 2, 3 and 4 and removing the hit on layer 3 . The results of the measurement are shown in Figure 6 for layer 2 (left) and layer 3 (right) modules. The hit resolution along the local- $x$ direction, that corresponds to the direction along which the pixel cell measures $100 \mu \mathrm{m}$, is found to be $\sigma_{2}=13.34 \pm 1.19 \mu \mathrm{m}$ for layer 2 and $\sigma_{3}=14.68 \pm 1.69 \mu \mathrm{m}$ for layer 3 . The two results are compatible with each other within the uncertainties, and the layer 2 resolution is compatible with the one of the old pixel detector, as expected. 

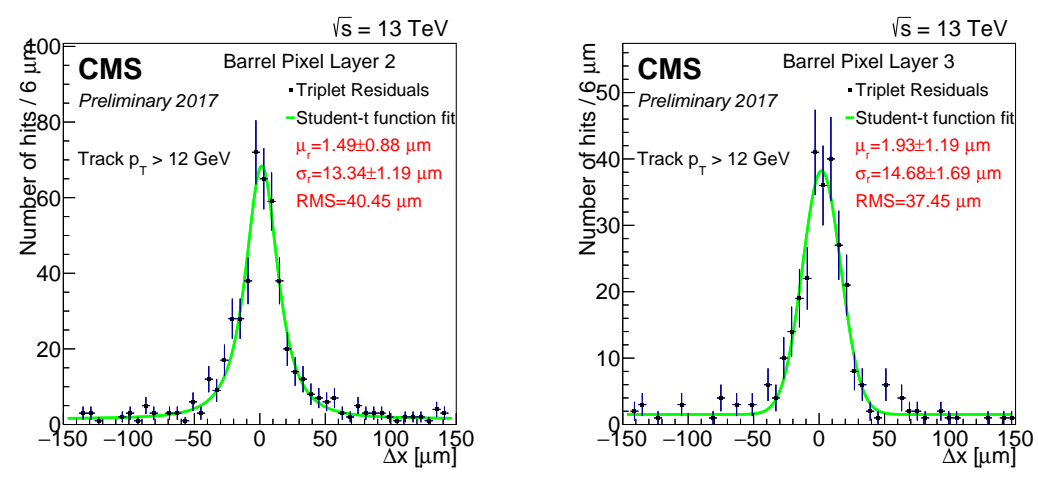

Figure 6: Distribution of residuals used to derive the hit resolution using the triplet method described in the text. The distributions for BPIX modules of layer 2 (left) and layer 3 (right) along the local- $x$ direction are shown [9].

\section{Summary}

The installation of the Phase-1 upgraded CMS pixel detector was successfully completed at the beginning of 2017. The new pixel detector could be precisely aligned down to the level of single modules already before the start of the pp collison data taking, using cosmic ray tracks. The first measurements of the pixel hit resolution performed with early pp collision data are in line with the expectations.

\section{References}

[1] CMS Collaboration, The CMS Experiment at the CERN LHC, 2008 JINST 3 S08004, doi:10.1088/1748-0221/3/08/S08004

[2] CMS Collaboration, CMS Technical Design Report for the Pixel Detector Upgrade, CMS-TDR-011, CERN-LHCC-2012-016 (2012), http://cds.cern.ch/record/1481838

[3] CMS Collaboration, CMS Tracking POG Performance Plots For 2017 with PhaseI pixel detector, https://twiki.cern.ch/twiki/bin/view/CMSPublic/TrackingPOGPerformance2017MC

[4] CMS Collaboration, Performance of heavy flavour identification algorithms in proton-proton collisions at $13 \mathrm{TeV}$ at the CMS experiment, 2017, CMS-DP-2017-012, http://cds.cern.ch/record/2263801

[5] CMS Collaboration, Alignment of the CMS silicon tracker during commissioning with cosmic rays, 2010 JINST 5 T03009, doi:10.1088/1748-0221/5/03/T03009

[6] CMS Collaboration, Alignment of the CMS tracker with LHC and cosmic ray data, 2014 JINST 9 P06009, doi:10.1088/1748-0221/9/06/P06009

[7] CMS Collaboration, CMS Tracker Alignment Performance Results 2016, 2017, CMS-DP-2017-021, http://cds.cern.ch/record/2273267

[8] CMS Collaboration, CMS Tracker Alignment Performance Results Start-Up 2017, https://twiki.cern.ch/twiki/bin/view/CMSPublic/TkAlignmentPerformancePhaseIStartUp17

[9] CMS Collaboration, The Performance plots for Phase 1 Pixel Detector 2017, https://twiki.cern.ch/twiki/bin/view/CMSPublic/PixelOfflinePlots2017 